\title{
Transient hair loss in patients with chronic spontaneous urticaria treated with omalizumab
}

\author{
${ }^{1}$ Department of Dermatology, Bispebjerg Hospital, Copenhagen, Denmark \\ ${ }^{2}$ Center for Medical Research Methodology, Department of Biomedical Sciences, University of Copenhagen, Copenhagen, \\ Denmark
}

\section{Corresponding author}

Misbah Noshela Ghazanfar

Department of Dermatology

Bispebjerg Hospital

Bispebjerg Bakke 23

DK-2400 Copenhagen NV

Denmark

Phone: +45 53309691

E-mail: misbah.noshela.ghazanfar@regionh.dk

\section{Doi}

10.23822/EurAnnACI.1764-1489.08

\begin{abstract}
Summary
Omalizumab (anti-IgE) is used as add-on therapy for antihistamine refractory chronic urticaria patients. The most commonly reported adverse effects were headache, arthralgia, upper respiratory infections, fatigue, nausea and injection-site reactions. However, lately a few cases of hair loss have been reported. We describe a case of transient hair loss in a young female patient after initiating treatment with omalizumab. Despite this side effect, the patient continued with omalizumab treatment for 10 months with good effect.
\end{abstract}

Dear Editor,

We read with interest the recent case report by Konstantinou et al. (1) published in your journal about the use of omalizum$\mathrm{ab}$ in three female patients with chronic spontaneous urticaria (CSU) who reported transient hair loss. In the case report by Konstantinou et al. (1) all three patients had previously been treated with non-sedating antihistamines, ranitidine and montelukast without any symptom relief. Furthermore, all of the patients were also treated with at least one short course of prednisolone due to severe angioedema and pruritus in the last four weeks before initiating treatment with omalizumab. Two of the female patients had no other known significant illnesses, whereas one of the patients was known with Hashimoto's thyroiditis since the past 12 months. All three patients reported transient hair loss after initiating treatment with omalizumab, however only one of the patients (with Hashimoto's thyroiditis) had visible alopecia areata. None of the patients discontinued their treatment.
Transient hair loss is not a commonly reported side effect of omalizumab. In the following we describe a similar case of a female patient from our dermatology department, who was treated with omalizumab and also experienced transient hair loss. The patient was a 27 -year-old woman with a three week history of urticaria who was referred to our department in June 2015. At the time of the referral, she had abdominal pain and she reported a recent urinary tract infection, eye infection and orolabial herpes infection. Urine and routine blood tests were normal aside from CRP, which was elevated to $53 \mathrm{mg} / \mathrm{l}$ (nor$\mathrm{mal}<10 \mathrm{mg} / \mathrm{l})$. The patient suffered from diffuse urticaria and swelling, primarily of her palms, soles and around her eyes. She was treated with non-sedating antihistamines four times daily as well as $50 \mathrm{mg}$ prednisolone daily for three days with some symptomatic relief. However, a few days later her symptoms worsened and she was re-hospitalized. Routine blood tests showed elevation of CRP to $33 \mathrm{mg} / \mathrm{l}$ and leukocytes to $10.7 \mathrm{x}$ $10^{9} / 1$ (normal 3.5-8.8 $\left.\times 10^{9} / 1\right)$. Chest X-ray, throat culture and 
urine sample and an abdominal ultrasound were all normal. A skin biopsy was also performed and it confirmed urticaria histologically with eosinophil as well as neutrophil infiltrates. The patient was treated with non-sedating antihistamines (fexofenadine $180 \mathrm{mg}$ ) four times daily and $50 \mathrm{mg}$ prednisolone daily without relief. Montelukast $10 \mathrm{mg}$ was added without any improvement. After three days with continuous aggravation of her urticaria rashes, omalizumab $300 \mathrm{mg}$ was initiated with fast reduction in symptoms and she was discharged the following day. Prednisolone was tapered to $5 \mathrm{mg}$ daily and due to recurrence of urticaria symptoms and development of concomitant joint pain and flu-like symptoms dapsone $100 \mathrm{mg}$ daily was added and omalizumab was increased to $300 \mathrm{mg}$ every second week, whereas antihistamines, montelukast and prednisolone were discontinued. After two weeks the patient was symptom free and she discontinued dapsone, whereas omalizumab was decreased to $300 \mathrm{mg}$ every fourth week. After three months of treatment, the patient reported shedding about $1 / 3$ of her scalp hair. There was no visible alopecia areata. The patient continued with omalizumab treatment and scored zero on the urticaria activity score in the past week (UAS7). After two months, the patient reported regrowth of her hair. The patient was completely symptom free; therefore the dosing interval of omalizumab was prolonged from four weeks to five weeks and then to six weeks. The patient discontinued treatment with omalizumab after 10 months as she was completely asymptomatic (UAS7 $=0$ ). However, the patient experienced flare up shortly after and was retreated with $300 \mathrm{mg}$ omalizumab every sixth weeks. Interestingly, the patient experienced no hair loss during this round of treatment or any other adverse effects.

We agree with Konstantinou et al. (1) that hair loss could be a transient side effect of omalizumab seen among some CSU patients. Particularly, since mast cells have been shown to be involved in the hair cycle (2), manipulation of mast cell activity could be speculated to account for this. However, the associ- ation with omalizumab is uncertain, as the patient described by Konstantinou et al. (1) with visible alopecia areata also had Hashimoto's thyroiditis, which is commonly associated with hair loss (3). Likewise, the hair loss in our case could have been caused by urticaria itself or the infectious diseases prior to urticaria. Also, our patient, and the cases described by Konstantinou et al. (1) were all treated with prednisolone, which has also been associated with hair loss. Furthermore, it is worth noting that hair loss is not a reported adverse effect in clinical phase trials of CSU and omalizumab. The most commonly reported adverse effects were headache, arthralgia, upper respiratory infections, fatigue, nausea and injection-site reactions (4-6). We conclude that hair loss may have multiple causes and that these must be accommodated in the explanation of the possible association between omalizumab use and hair loss in patients with urticaria.

\section{References}

1. Konstantinou G. N., Chioti A. G., Danilidis M. Self-reported hair loss in patients with chronic spontaneous urticaria treated with omalizumab: an under-reported, transient side effect? Eur Ann Allergy Clin Immunol. 2016;48:205-7.

2. Paus R., Peters E., Eichmiiller $S$ et al. Neural Mechanisms of Hair Growth Control. JID Symposium Proceedings. 1997;2:61-8.

3. Thomas E. A., Kadyan R. S. Alopecia areata and autoimmunity: a clinical study. Indian J Dermatol. 2008;53:70-4.

4. Saini S., Bindslev-Jensen C., Maurer M. et al. Efficacy and safety of omalizumab in patients with chronic idiopathic / spontaneous urticaria who remain symptomatic on $\mathrm{H}_{1}$ antihistamines: a randomized, placebo-controlled study. J Clin Investig Dermatol. 2015; 135:67-75.

5. Saini S, Rosen K. E., Hsieh H.-J. et al. A randomized, placebo-controlled, dose-ranging study of single-dose omalizumab in patients with $\mathrm{H} 1$-antihistamine-refractory chronic idiopathic urticaria. J Allergy Clin Immunol Pract. 2011;128:567-73.

6. Maurer M, Altrichter S., Bieber T et al. Efficacy and safety of omalizumab in patients with chronic urticaria who exhibit IgE against thyroperoxidase. J Allergy Clin Immunol. 2011;128:202-9. 